\title{
YgfZ contributes to secretion of cytotoxic necrotizing factor 1 into outer-membrane vesicles in Escherichia coli
}

Correspondence

Kwang Sik Kim

kwangkim@jhmi.edu

Received 18 August 2011

Revised 1 November 2011

Accepted 12 December 2011

\author{
Hao Yu and Kwang Sik Kim
}

Division of Pediatric Infectious Diseases, Johns Hopkins University School of Medicine, 200 North Wolfe Street, Room 3157, Baltimore, MD 21287, USA

\begin{abstract}
Cytotoxic necrotizing factor 1 (CNF1), a Rho GTPase-activating bacterial toxin, has been shown to contribute to invasion by meningitis-causing Escherichia coli $\mathrm{K} 1$ of human brain microvascular endothelial cells (HBMEC), which constitute the blood-brain barrier. However, CNF1 is a cytosolic protein and it remains unclear how its secretion occurs, contributing to $E$. coli invasion of HBMEC. To investigate the genetic requirement for CNF1 secretion in E. coli K1 strain RS218, we performed mini-Tn5 in vitro mutagenesis and constructed a transposon mutant library of strain $\mathrm{NBC}$, in which $\beta$-lactamase was fused to the C-terminus of CNF1 in the chromosome of strain RS218. We identified a transposon mutant (NBC-1E6) that exhibited reduced $\beta$-lactamase activity in its culture supernatant and had the transposon inserted into the $y g f Z$ gene. When $y g f Z$ was deleted from the genome of strain RS218 ( $\Delta y g f Z$ ), the translocation of CNF1 into HBMEC was impaired. Subcellular localization analysis of CNF1 demonstrated that YgfZ, a periplasmic protein, contributes to secretion of CNF1 into outer-membrane vesicles (OMVs). The $\Delta y g f Z$ mutant was significantly defective in invasion of HBMEC compared to the parent $E$. coli $\mathrm{K} 1$ strain. The defects of the $\triangle y g f Z$ mutant in CNF1 secretion into OMVs and translocation into HBMEC as well as invasion of HBMEC were abrogated by complementation with ygfZ. Taken together, our findings demonstrate that $\mathrm{YgfZ}$ contributes to CNF1 secretion into OMVs in meningitis-causing $E$. coli $\mathrm{K} 1$.
\end{abstract}

\section{INTRODUCTION}

CNF1, the paradigm of the Rho GTPase-activating bacterial toxins (Boquet, 2001; Knust \& Schmidt, 2010; Lemonnier et al., 2007), is frequently associated with uropathogenic and meningitis-causing Escherichia coli strains (Khan et al., 2002; Landraud, et al., 2000). CNF1 activates Rho GTPases by deamidation of glutamine 63, converting it into glutamic acid, thus inhibiting GTPhydrolysing activity, resulting in constitutive activation of Rho GTPase. CNF1 has been shown to contribute to polymerization of F-actin, increased formation of stress fibres and phagocytosis (Caprioli et al., 1983; Falzano et al., 1993; Flatau et al., 1997; Schmidt et al., 1997; VouretCraviari et al., 1999). CNF1-like toxins have also been found in other bacteria, including the dermonecrotic toxin of Bordetella spp. and the CNF $\gamma$ from Yersinia pseudotuberculosis (Knust \& Schmidt, 2010; Kume et al., 1986; Lockman et al., 2002).

We have shown that CNF1 contributes to invasion by $E$. coli K1 of human brain microvascular endothelial cells

Abbreviations: AP, alkaline phosphatase; Bla, $\beta$-lactamase; HBMEC, human brain microvascular endothelial cells; CNF1, cytotoxic necrotizing factor 1; OMV, outer-membrane vesicle.
(HBMEC) and penetration into the brain via the interaction with its receptor, 37 laminin receptor precursor (37LRP)/67 laminin receptor (67LR) (Chung et al., 2003; Khan et al., 2002; Kim et al., 2005). CNF1 is a cytoplasmic protein and its secretion is, therefore, a strategy utilized by meningitis-causing E. coli $\mathrm{K} 1$ to invade the blood-brain barrier (Khan et al., 2002; Kim, 2003, 2008). However, it remains incompletely understood how CNF1 secretion occurs. No typical signal peptide is found in the CNF1 sequence. Recent studies have shown that CNF1 is transported to the culture supernatant in a complex with outer-membrane vesicles (OMVs) in uropathogenic E. coli strains J96 and CP9 (Davis et al., 2006; Kouokam et al., 2006).

OMVs from a number of bacterial species have been shown to contain virulence factors, some exhibiting immunomodulatory effects, and others exhibiting adherence to and intoxication of host cells (Mashburn-Warren \& Whiteley, 2006). Several studies suggest that the secretion of cargo proteins into OMVs is a regulated process. For example, in enterotoxigenic E. coli, delivery of toxins via OMVs has been shown to be associated with host cells in a time- and receptor-dependent manner (Kesty et al., 2004), suggesting that OMVs serve as transporters mediating entry of 
bacterial virulence factors into host cells. In Pseudomonas aeruginosa, interaction of quorum signals with outermembrane lipids was shown to be required for OMV formation (Mashburn-Warren et al., 2008). However, it is unclear whether delivery of toxins and other bacterial molecules into target cells from those membrane blebs can be regarded as true secretion, because there was no evidence of the involvement of 'secretion' factors independent of membrane perturbation (Economou et al., 2006).

In order to study the genetic requirements for secretion of CNF1 in meningitis-causing E. coli $\mathrm{K} 1$, we designed a Tn5 mutational screening strategy by applying TEM $\beta$-lactamase as the reporter to monitor CNF1 secretion. We demonstrated that $\mathrm{YgfZ}$, a periplasmic protein, contributes to secretion of CNF1 into OMVs.

\section{METHODS}

Bacterial strains, plasmids and growth conditions. The bacterial strains and plasmids used are shown in Table 1. E. coli K1 strain
RS218 (O18: K1:H7) is a cerebrospinal fluid isolate from a neonate with meningitis (Khan et al., 2002). Strain NBC was used for Tn5 mutagenesis, in which the $\beta$-lactamase reporter gene was translationally fused to the C-terminus of the cnfl gene in the chromosome of strain RS218, as previously described (Yu \& Kim, 2010). E. coli K-12 strain DH5 $\alpha$ was used as the host for plasmids, and EC100D pirl16+ (Epicentre Biotechnologies) as the host for R6k $\gamma$ origin plasmid. E. coli strains were routinely grown at $37{ }^{\circ} \mathrm{C}$ in Luria Broth (LB). Where appropriate, the medium was supplemented with ampicillin $(100 \mu \mathrm{g}$ $\left.\mathrm{ml}^{-1}\right)$, spectinomycin $\left(100 \mu \mathrm{g} \mathrm{ml}^{-1}\right)$, tetracycline $\left(10 \mu \mathrm{g} \mathrm{ml}^{-1}\right)$ or chloramphenicol $\left(20 \mu \mathrm{g} \mathrm{ml}^{-1}\right)$.

Transposome formation and transposition mutagenesis. These were performed as described previously (Geddes et al., 2005; Yu \& Kim, 2010). Briefly, the transposome complex was formed by incubating Tn5 transposome SR and hyperactive Tn5 transposase (Epicentre Biotechnologies) for $1 \mathrm{~h}$ at $37^{\circ} \mathrm{C}$. Transposomes were electroporated into competent NBC cells. Transposon insertion mutants were selected with spectinomycin.

$\boldsymbol{\beta}$-Lactamase (Bla) activity assay. Bla activity was determined as described previously (Yu \& Kim, 2010). Briefly, nitrocefin at a final concentration of $0.1 \mathrm{mM}$ was incubated with bacterial culture supernatant (obtained by centrifugation at $4000 \mathrm{~g}$ for $10 \mathrm{~min}$ ) for

Table 1. Strains and plasmids

\begin{tabular}{|c|c|c|}
\hline Strain or plasmid & Relevant characteristic(s) & Reference or source \\
\hline \multicolumn{3}{|l|}{ E. coli strains } \\
\hline RS218 & $\begin{array}{l}\text { O18: K1:H7 strain isolated from the cerebrospinal fluid of a neonate with } E \text {. coli } \\
\text { meningitis }\end{array}$ & Khan et al. (2002) \\
\hline EC100D & $\begin{array}{l}\mathrm{F}^{-} \text {mcrA } \Delta(\text { mrr-hsdRMS-mcrBC) } \phi 80 \mathrm{~d} l a c Z \Delta \mathrm{M} 15 \Delta \text { lacX74 recA1 endA1 araD139 } \\
\Delta(\text { ara, leu }) 7697 \text { galU galK } \lambda^{-} \text {rpsL nupG } \text { pir }^{+}(\mathrm{DHFR})\end{array}$ & Epicentre Biotechnologies \\
\hline DH5 $\alpha$ & $\begin{array}{l}\mathrm{F}^{\prime} \phi 80 \mathrm{~d} l a c Z \Delta \mathrm{M} 15 \Delta(\text { lacZYA-argF }) U 169 \text { deoR recAl endA1 hsdR17 }\left(\mathrm{r}_{\mathrm{k}}^{-} \mathrm{m}_{\mathrm{k}}^{+}\right) \text {phoA } \\
\text { supE44 } \lambda^{-} \text {thi-1 }\end{array}$ & Lab stock \\
\hline NBC & $\begin{array}{l}\beta \text {-Lactamase reporter gene translationally fused to the C-terminus of the cnfl gene } \\
\text { in the chromosome of strain RS } 218\end{array}$ & Yu \& Kim (2010) \\
\hline NBC-1E6 & NBC with Tn5 inserted in the $y g f Z$ gene & This study \\
\hline YK6 & RS218 (O18: K1:H7) ygfZ deletion mutant & This study \\
\hline HNS & RS218 (O18: K1:H7) hns deletion mutant & Lab stock \\
\hline YK7 & $\begin{array}{l}\text { RS218 (O18: K1:H7) } y g f Z \text { deletion mutant complemented with } y g f Z \text { under the control } \\
\text { of its native promoter }\end{array}$ & This study \\
\hline YK14 & NBC-1E6 complemented with $y g f Z$ under the control of its native promoter & This study \\
\hline YK15 & RS218 (O18:K1:H7) ygfZ and hns double deletion mutant & This study \\
\hline YK16 & $\begin{array}{l}\text { RS218 (O18: K1:H7) ygfZ and hns double deletion mutant complemented with } y g f Z \\
\text { under the control of its native promoter }\end{array}$ & This study \\
\hline \multicolumn{3}{|c|}{ 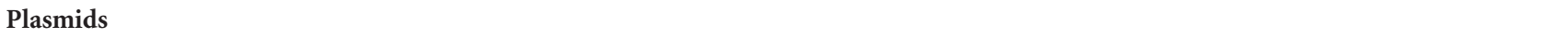 } \\
\hline pFLAG-CTC & $\begin{array}{l}5348 \text { bp E. coli expression vector used for cytoplasmic expression of a properly } \\
\text { inserted ORF as a C-terminal FLAG fusion protein }\end{array}$ & Sigma \\
\hline pFLAG-CTS-BAP & $\begin{array}{l}\text { E. coli plasmid used for efficient and controlled periplasmic expression of C-terminal } \\
\text { FLAG-BAP fusion protein }\end{array}$ & Sigma \\
\hline pCTC-YgfZ & Expression vector of YgfZ fused with FLAG at the C-terminus & This study \\
\hline $\mathrm{pCXN}$ & CNF1 coding region cloned into the KpnI site of pCX340, tetracycline resistant & Yu \& Kim (2010) \\
\hline pKD3 & Containing chromphenicol-resistance gene and $\mathrm{R} 6 \mathrm{k} \gamma$ replication origin & Datsenko \& Wanner (2000) \\
\hline pKD47 & $\begin{array}{l}\text { Derivative of pKD46 (Datsenko \& Wanner, 2000), with the only modification that } \\
\text { blaM was replaced by spectinomycin-resistance gene }\end{array}$ & This study \\
\hline pGRG36 & $\operatorname{Tn} 7$ insertion vector, ampicillin resistance, temperature sensitive & McKenzie \& Craig (2006) \\
\hline pSR & Tn 5 vector, spectinomycin resistant, $\mathrm{R} 6 \mathrm{k} \gamma$ replication origin & Yu \& Kim (2010) \\
\hline pG- $y g f Z$ & $\begin{array}{l}\text { DNA fragment containing } y g f Z \text { gene obtained by PCR amplification from genomic } \\
\text { DNA, and ligated into AvrII and PacI sites of pGRG36 }\end{array}$ & This study \\
\hline
\end{tabular}


$20 \mathrm{~h}$. Nitrocefin, as the chromogenic substrate of Bla, undergoes a distinctive colour change from yellow $\left(\lambda_{\max } 390 \mathrm{~nm}\right.$ at $\left.\mathrm{pH} 7.0\right)$ to red $\left(\lambda_{\max } 486 \mathrm{~nm}\right.$ at $\left.\mathrm{pH} 7.0\right)$ as the amide bond in the $\beta$-lactam ring is hydrolysed by Bla. Bla activity was read as positive if the colour change to red occurred. Spectrophotometric assays for Bla using nitrocefin were also carried out by measuring changes in $A_{486}$.

Genomic DNA isolation and sequencing. Genomic DNA was isolated from Tn5 mutants. Quantity of chromosomal DNA was measured with the Quant-iT dsDNA BR assay kit (Invitrogen); $12 \mu \mathrm{l}$ genomic DNA $\left(0.5 \mu \mathrm{g} \mathrm{l}^{-1}\right)$ and $12 \mu \mathrm{l}$ sequencing primer SR-Seq $(8 \mu \mathrm{M})$ were sent to the DNA Synthesis and Sequencing Facility, Johns Hopkins University School of Medicine, for sequencing.

ygfZ gene deletion and complementation. To delete the $y g f Z$ gene, a chloramphenicol-resistance cassette was amplified from $\mathrm{pKD} 3$ using primers 1E6-KOF and 1E6-KOR (Table 2). The PCR product was inserted into the chromosome by Lambda Red-mediated allele replacement (Datsenko \& Wanner, 2000). The correct insertion was verified by PCR with primers 1E6CKF and 1E6CKR (Table 2).

For gene complementation, $\operatorname{Tn} 7$ site-specific insertion of the $y g f Z$ gene into the second benign site in the chromosome of the mutant was carried out as described previously (McKenzie \& Craig, 2006; Yu \& Kim, 2010). Briefly, the $y g f Z$ gene together with its native promoter was amplified from the genomic DNA of strain RS218 by primers 1E6 s and 1E6-a (Table 2), and then ligated into AvrII and PacI sites of pGRG36. The resulting plasmid was designated pG- $y g f Z$. pG- $y g f Z$ was then electroporated into NBC-1E6 (Tn5::ygfZ), the $\Delta y g f Z$ mutant, or $\Delta y g f Z / \Delta h n s$, and transformants were selected on LB plates containing ampicillin at $32{ }^{\circ} \mathrm{C}$. The insertion of $\mathrm{Tn} 7$ in the attachment site was verified by PCR with primers Tn7-ckf and Tn7-ckr (Table 2) (Fig. 1c).

Assessment of CNF1 translocation into HBMEC. The CNF1 translocation assay was done as described previously (Mills et al., 2008; Yu \& Kim, 2010). Briefly, HBMEC grown in monolayers were preloaded with CCF4/AM dye and incubated with bacteria harbouring pCXN. The expression of the CNF1-Bla fusion from pCXN was induced with $1 \mathrm{mM}$ IPTG. Upon entry into HBMEC, the nonfluorescent esterified CCF4/AM substrate is converted to fluorescent green CCF4 by cellular esterases. Translocation of CNF1-Bla into HBMEC induces catalytic cleavage of the CCF $4 \beta$-lactam ring, which produces an easily detectable colour change in CCF4 fluorescence from green to blue emission (Charpentier \& Oswald, 2004; Yu \& Kim, 2010; Zlokarnik et al., 1998). After $45 \mathrm{~min}$ of infection, the translocation of CNF1-Bla hybrid into HBMEC was observed with a Nikon fluorescence microscope.

Plasmid construction. To subclone $y g f Z$ into the FLAG-tagged expression vector pFLAG-CTC (Sigma), the $y g f Z$ coding region was amplified from RS218 genomic DNA with primers 1E6-s2 and 1E6-a2 (Table 2), and the PCR product was ligated into the NdeI and SalI sites of pFLAG-CTC, resulting in plasmid pCTC-YgfZ.

Cell fractionation. Cytoplasmic, periplasmic and outer-membrane fractions were obtained by the osmotic shock method as described previously (Wai et al., 2003; Yu \& Kim, 2010). Periplasmic suspension was filtered through a $0.22 \mu \mathrm{m}$ filter to remove any residual bacterial cells, and then precipitated by the sodium deoxycholate/trichloroacetic acid method ( $\mathrm{Yu}$ \& Kim, 2010). OMVs were isolated by ultracentrifugation (100000 $\mathrm{g}$ for $2 \mathrm{~h}$; SW41Ti rotor, Beckman) as described previously (Kouokam et al., 2006).

Western blot assays. Cell fractions were separated by SDS-PAGE, and then protein samples were transferred to nitrocellulose membrane. The blots were blocked with $5 \%$ skim milk in TBS $\left(25 \mathrm{mM}\right.$ Tris, $\mathrm{pH} 7.4,150 \mathrm{mM} \mathrm{NaCl}$ ) for $60 \mathrm{~min}$ at $22^{\circ} \mathrm{C}$. The membrane was then incubated for $2 \mathrm{~h}$ at $22{ }^{\circ} \mathrm{C}$ with primary antibody. Primary antibodies used in this study were anti-CNF1 monoclonal antibody (DD1) (Meysick et al., 2001), alkaline phosphatase (AP or PhoA) monoclonal antibody (Millipore), $\beta$-Gal polyclonal antibody (Millipore), NuoCD polyclonal antibody (Castro-Guerrero et al., 2010), anti-FLAG BioM2 monoclonal antibody (Sigma) and OmpA monoclonal antibody (Yu \& Kim, 2010). The membrane was washed with $0.5 \%$ Tween 20 in TBS and subsequently incubated for $60 \mathrm{~min}$ at room temperature with horseradish-peroxidase-linked secondary antibodies. The membrane was developed with Amersham ECL Advance (for detection of CNF1, $\beta$-Gal and NuoCD) or the Amersham ECL detection system (for detection of FLAG, AP and OmpA) (GE Healthcare).

E. coli invasion of HBMEC. E. coli invasion assays were performed in HBMEC monolayers as previously described (Khan et al., 2002; Yu \& Kim, 2010). Briefly, confluent HBMEC grown in 24-well plates were incubated with $10^{7}$ c.f.u. of E. coli (m.o.i. 100) in experimental medium. The plates were incubated for $90 \mathrm{~min}$ at $37{ }^{\circ} \mathrm{C}$ in $5 \% \mathrm{CO}_{2}$ to allow bacterial invasion to occur. The number of intracellular bacteria was determined by culturing on blood agar plates after killing extracellular bacteria by incubation of the HBMEC monolayer with experimental medium containing gentamicin $\left(100 \mu \mathrm{g} \mathrm{ml}^{-1}\right)$ for $1 \mathrm{~h}$.

Table 2. Primers

\begin{tabular}{|c|c|}
\hline Primer & Sequence $\left(5^{\prime}-3^{\prime}\right)^{*}$ \\
\hline 1E6-KOF & TCTTTTACCATTAGTTATTAATACGCCGTTAAGCAACTCAGGACTCTATTGTGTAGGCTGGAGCTGCTTC \\
\hline 1E6-KOR & GACACACCAGTGTCGCATCAGGCGATAAATGCCTGATGCGATACAGAGATCATATGAATATCCTCCTTAG \\
\hline 1E6CKF & GACGCTCTATCACGCCATTCC \\
\hline 1E6CKR & GGGCAACTGTTCGTGGGTCT \\
\hline 1E6-a & CCACGATCGCGATACAGAGATTTACTCTTC \\
\hline $1 \mathrm{E} 6 \mathrm{~s}$ & CCAGCCTAGGTGTCTCACGAAAATCCAGTA \\
\hline $1 \mathrm{E} 6-\mathrm{s} 2$ & ACCTGTCATATGGCTTTTACACCTTTTCC \\
\hline 1E6-a2 & GCCAGTCGACCTCTTCCAGCGAATATGGC \\
\hline Spc-SeqR & GCCTTGCTGTTCTTCTACGG \\
\hline Tn7-ckf & ACGGTCGGGAACTGGAAC \\
\hline Tn7-ckr & TGACCAGCCGCGTAACCT \\
\hline
\end{tabular}

${ }^{\star}$ Restriction sites for cloning are underlined. 

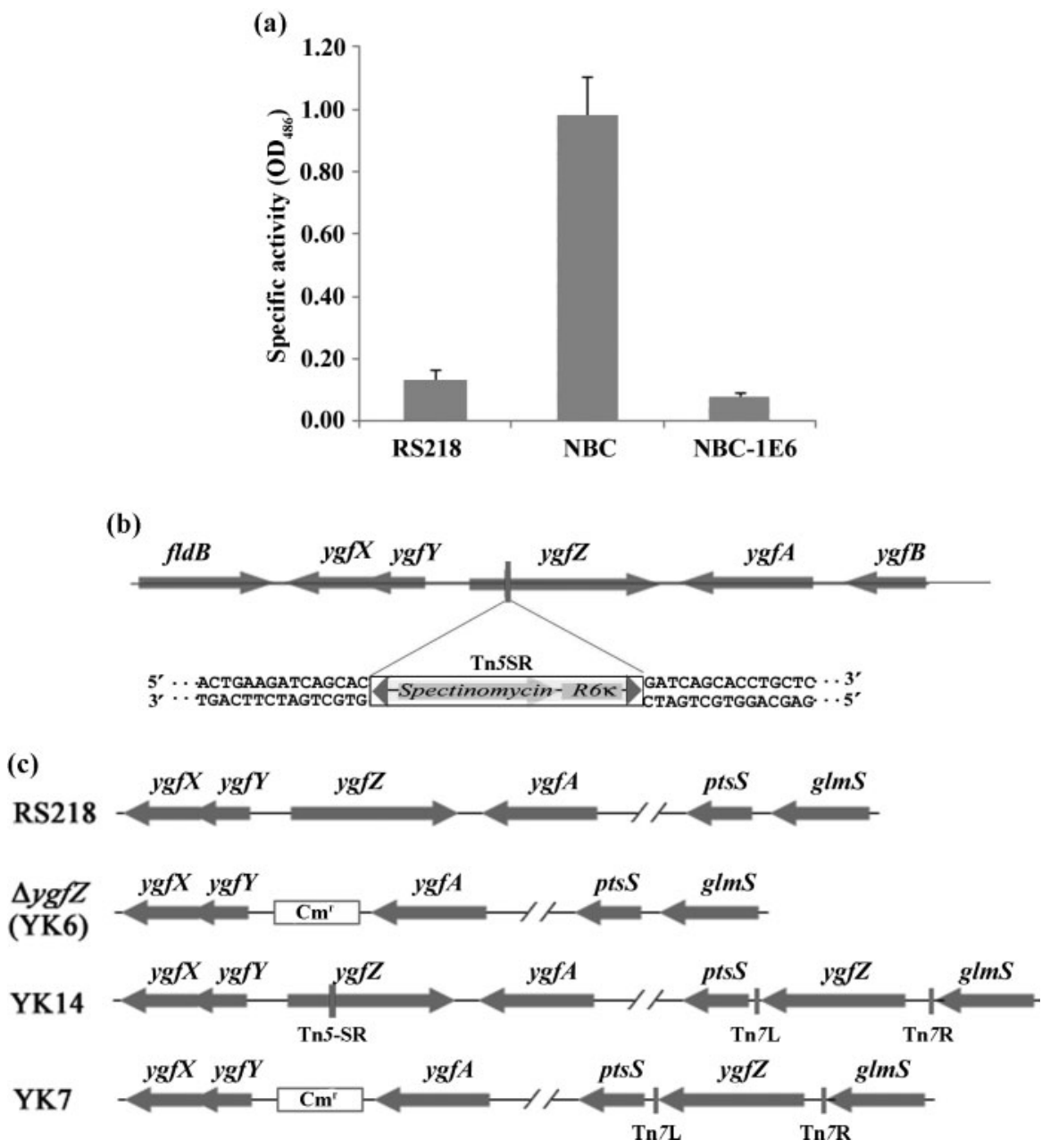

Fig. 1. Identification of the $y g f Z$ gene as a genetic requirement for CNF1 secretion. (a) Bla activity in the culture supernatant of strains RS218, NBC and NBC-1E6 (Tn5::ygfZ). Bacteria were grown overnight in brain-heart infusion broth (static at $37{ }^{\circ} \mathrm{C}$ ), the culture supernatant was obtained by centrifugation at $5000 \mathrm{~g}$ for $10 \mathrm{~min}$, and Bla activity was determined based on the $A_{486}$. The Bla activity shown represents the mean \pm SD of results from three experiments in triplicate. (b) Transposon insertion within the ygfZ gene in the mutant strain NBC-1E6 (Tn5::ygfZ). (c) Schematic representation of the chromosomal structure of ygfZ mutants constructed in this study. $\mathrm{Cm}^{r}$, chloramphenicol resistance gene. YK6 is the ygfZ deletion mutant; YK7 is a complemented strain of the $y g f Z$ deletion mutant with ygfZ; YK14 was derived from NBC-1E6 by complementing the $y g f Z$ gene in the Tn7 attachment site. YK15 and YK16 are not listed in this figure; they represent the ygfZ deletion mutant and the complemented strain, respectively (i.e. identical to YK6 and YK7, respectively), in the background of $h n s$ deletion.

Assays were performed in triplicate and repeated at least three times. Results were expressed as relative invasion frequencies (percentage invasion compared to that of the parent strain, RS218).

\section{RESULTS}

\section{Tn5 mutagenesis screen identifies the ygfZ gene}

To investigate the genetic requirement for CNF1 secretion in strain RS218, we performed mini-Tn5 in vitro mutagenesis and constructed a mutant library of strain NBC, in which $\beta$ lactamase (Bla) was fused to the C-terminus of CNF1 in the chromosome of strain RS218. In strain NBC, Bla secretion is entirely dependent on the CNF1 secretion machinery (Yu \& Kim, 2010), and the secretion of CNF1-Bla fusion protein into the culture supernatant was monitored by measuring the Bla activity. For Bla assay, strain NBC was used as a positive control, and strain RS218 was used as a negative control. We identified a mutant (NBC-1E6) that exhibited barely detectable Bla activity based on both visual colour change and the spectrometric reading. The mutant was significantly defective in secretion of CNF1 into the culture medium, based on the Bla activity (Fig. 1a). We determined the location of the transposon insertion as being within the $y g f Z$ gene by direct DNA sequencing of the mutant's genomic DNA (Fig. 1b). 


\section{CNF1 translocation is impaired in ygfZ deletion mutant}

We next deleted the entire $y g f Z$ gene from the chromosome of strain RS218, and examined the ability of the resulting strain to translocate CNF1 into HBMEC using CNF1-Bla fusion protein expressed from plasmid pCXN, as previously described (Yu \& Kim, 2010). In pCXN, CNF1 was translationally fused with Bla, and controlled by the trc promoter. E. coli transformants harbouring pCXN were pre-induced with $1 \mathrm{mM}$ IPTG and then added to HBMEC pre-loaded with CCF4/AM dye. After 45 min incubation, translocation of CNF1 into HBMEC was visualized by fluorescence microscopy. As expected, wild-type strain RS218 exhibited successful translocation of CNF1-Bla protein into HBMEC, as shown by the emission of bright blue fluorescence (Fig. 2). In screening a $\times 10$ view of the HBMEC monolayer, 147 bright blue cells were observed compared to 332 green cells. However, the translocation of CNF1-Bla into HBMEC was impaired in strain $\triangle y g f Z$, as only 6 weakly blue cells were observed, compared to 445 green cells (Fig. 2). The translocation of CNF1-Bla fusion into HBMEC in strain $\Delta y g f Z$ was, however, restored in the complemented strain YK7 ( $y y g f Z:: y g f Z)$, which demonstrated 163 blue cells and 392 green cells (Figs $1 \mathrm{c}$ and 2).

\section{YgfZ contributes to secretion of CNF1 into OMVs}

In order to determine the steps that are involved in defective CNF1 secretion in strain $\Delta y g f Z$, we compared the subcellular distribution of CNF1 between the $\Delta y g f Z$ mutant and wild-type strain RS218. The amounts of CNF1 in the cytoplasmic and periplasmic fractions were similar between RS218 and the $\Delta y g f Z$ mutant (Fig. 3a), suggesting that the defect in CNF1 translocation into HBMEC in the $\Delta y g f Z$ strain is likely to occur at the step of crossing the outer membrane from the periplasmic space. To test this possibility, we fractionated strains RS218, NBC, NBC-1E6 (Tn5:: $y g f Z$ ) and the genetically complemented strain YK14 (NBC-1E6 complemented with $y g f Z$ by Tn7 transposition, Fig. 1c). CNF1-Bla was present in the cytoplasmic, periplasmic and membrane fractions of strain NBC-1E6, but not in culture supernatant; in strain NBC and the complemented strain YK14, CNF1-Bla was present in all four fractions (Fig. 3b). The membrane fraction was not separated into the inner and outer membranes because $\mathrm{N}$-laurylsarcosine would denature CNF1-Bla fusion protein and interfere with the Bla activity. Since there is no detectable CNF1 in the inner membrane (Kouokam et al., 2006), the Bla activity detected in the membrane fraction is likely to represent the amount of CNF1 in the outermembrane fraction.

It has been shown that CNF1 is downregulated by H-NS (histone-like nucleoid structuring protein) in uropathogenic E. coli strain J96, and hns deletion led to increased CNF1 expression in this strain (Kouokam et al., 2006), suggesting that hns deletion is a useful tool for demonstrating CNF1 in cell fractions. We therefore examined CNF1 secretion in the H-NS deletion background of strain RS218. As indicated by markers of each fraction, the different cell fractions exhibited their expected protein profile. In the $\Delta h n s$ mutant, the presence of CNF1 was detected in the cytoplasmic, periplasmic, outer-membrane and OMV fractions (Fig. 3c, lanes 1). In contrast, in the $\Delta y g f Z / \Delta h n s$ double mutant, CNF1 was detected in the cytoplasmic, periplasmic and outer-membrane fractions, but not in the OMVs (Fig. 3c, lanes 2). It is important to

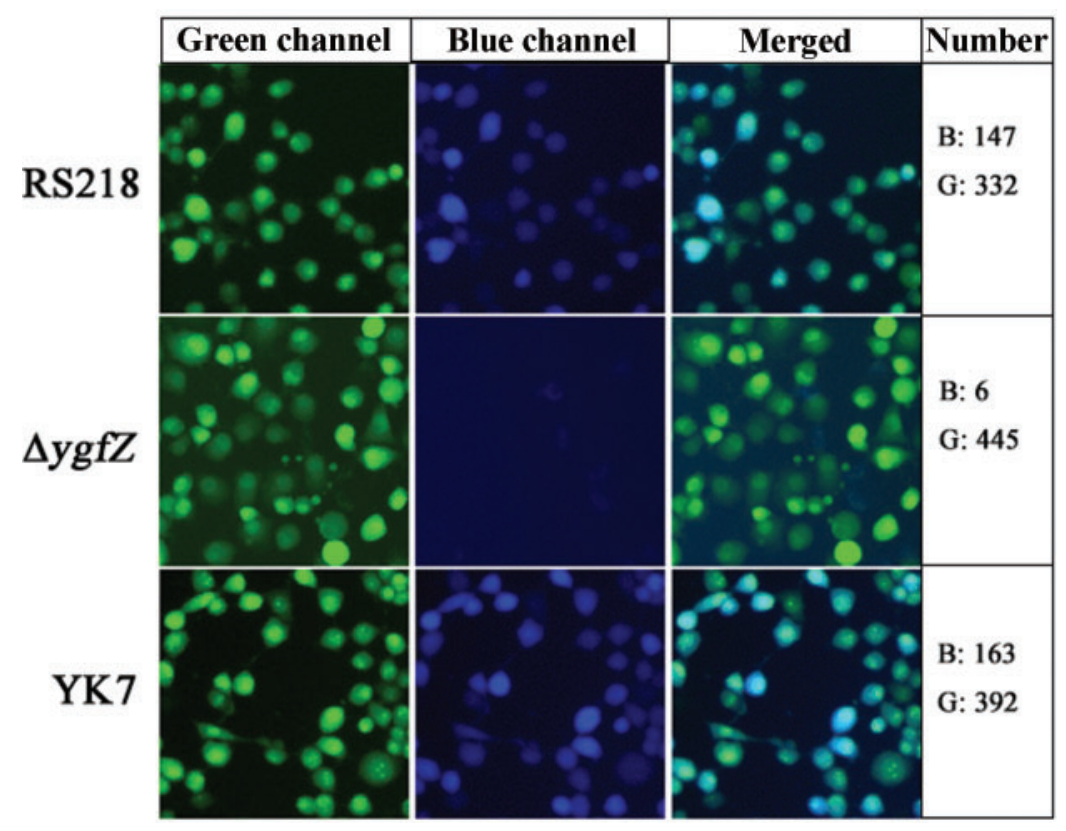

Fig. 2. Analysis of the translocation of CNF1 into HBMEC. HBMEC were pre-loaded with the Bla substrate CCF4/AM for $1 \mathrm{~h}$, and then infected with $E$. coli strains bearing plasmid pCXN. pCXN expresses the CNF1-Bla fusion. After 45 min incubation, the translocation of CNF1 into HBMEC, indicated by blue emission, was visualized by fluorescence microscopy. RS218 is the wild-type strain, $\Delta y g f Z$ is a $y g f Z$ deletion mutant, and YK7 is the $y g f Z$ deletion mutant complemented with $y g f Z$. The numbers of green $(G)$ and blue $(B)$ cells from a $\times 10$ view are listed on the right. 
(a)

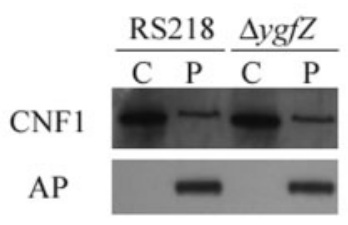

(c)

$$
\frac{\mathrm{C}}{12} \frac{\mathrm{P}}{1} 2 \frac{\mathrm{OM}}{1} \frac{2}{1} \frac{\mathrm{OMV}}{1}
$$

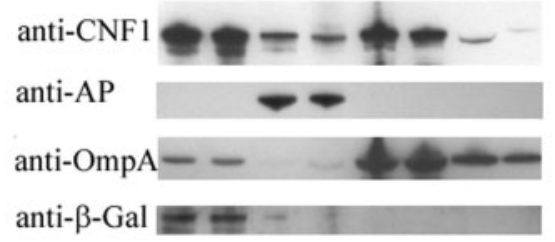

(b)

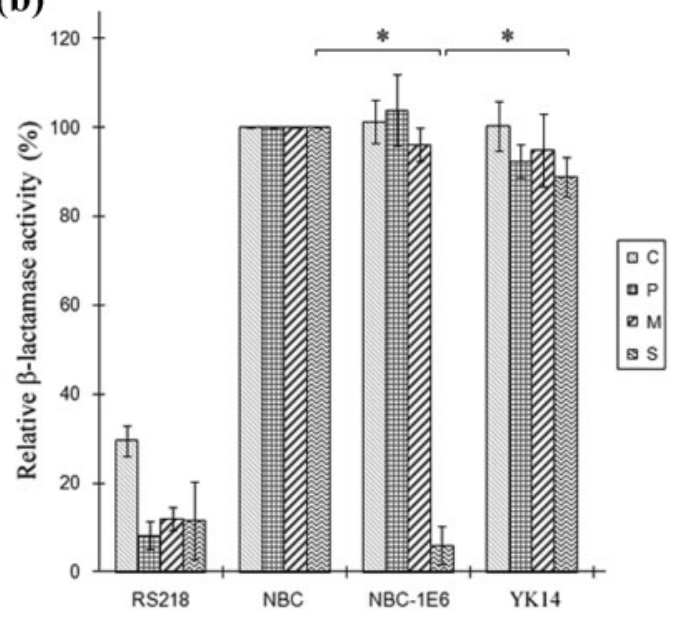

Fig. 3. YgfZ contributes to CNF1 secretion into OMVs. (a) Western blot analysis of periplasmic (P) and cytoplasmic (C) distribution of CNF1 in RS218 and $\Delta y g f Z$. Alkaline phosphatase (AP) was used as a marker for the periplasm. (b) Relative Bla activity in the cytoplasmic $(C)$, periplasmic $(P)$, membrane $(M)$ and culture supernatant $(S)$ fractions of strains RS218, NBC, NBC-1E6 (Tn5::ygfZ) and YK14 (NBC-1E6 complemented with ygfZ). Assays were performed in triplicate and repeated at least three times. Results (mean $\pm \mathrm{SD}$ ) are expressed as relative Bla activity (percentage activity compared to that of strain NBC, which was set at $100 \%)$. ${ }^{*}, P<0.05 \%$. (c) Western blot analysis of proteins from the cytoplasmic (C), periplasmic (P), outermembrane (OM) and OMV fractions from $\Delta h n s$ (lanes 1) and $\Delta h n s / \Delta y g f Z$ mutant (lanes 2). For cytoplasmic protein, $40 \mu g$ total cellular proteins from crude bacterial extracts were loaded in each well. Alkaline phosphatase (AP) and OmpA were used as the loading markers for periplasm and outer membrane (including OMVs), respectively. CNF1, AP, OmpA and $\beta$-Gal were detected by their respective specific antibodies, and different ECL substrates were used as described in Methods.

note that the amount of OmpA was similar between the OMVs isolated from $\Delta h n s$ and $y g f Z / \Delta h n s$ mutants (Fig. $3 c$ ), suggesting that the failure to demonstrate CNF1 in OMVs in the $\Delta y g f Z$ mutant is not generic to all proteins and/or related to technical issues of OMV preparation. The ability to demonstrate CNF1 in OMVs in the $\Delta y g f Z / \Delta h n s$ mutant was restored by complementation with $y g f Z$ containing its own promoter (strain YK16) (Fig. 4b). Taken together, these findings demonstrate that YgfZ is likely to be involved in CNF1 secretion into OMVs in E. coli K1.

It was of interest that SDS-PAGE-based comparison of the protein profiles of the OMVs isolated from the $\Delta h n s$ mutant, the $\Delta y g f Z l \Delta h n s$ mutant and the $\Delta y g f Z / \Delta h n s$ mutant complemented with $y g f Z$ revealed a different pattern of protein bands (Fig. 4a). The dominant protein in the band position of CNF1 was $\alpha$-haemolysin as revealed by mass spectrometry analysis, and the secretion of $\alpha$-haemolysin into OMVs was also affected when $y g f Z$ was deleted (Fig. 4a). $\alpha$-Haemolysin is a known protein secreted into OMVs, and $\alpha$-haemolysin associated with OMVs is more stable than free $\alpha$-haemolysin (Aldick et al., 2009). However, deletion of the type I secretion system responsible for the secretion of $\alpha$-haemolysin did not affect CNF1 secretion (data not shown), suggesting that different secretion systems are likely to be involved in secretion of $\alpha$-haemolysin and CNF1. YgfZ is, therefore, likely to affect a shared secretion machinery for selective loading of cargo proteins, including CNF1 and $\alpha$ haemolysin, into the OMVs. Taken together, our results demonstrate that secretion of CNF1 as well as $\alpha$ haemolysin into OMVs is diminished in the $\Delta y g f Z$ mutant, and it occurred without any detectable decrease of OmpA in OMVs.

\section{YgfZ is predominantly localized in the periplasmic space}

In order to understand how YgfZ affects CNF1 secretion, we cloned $y g f Z$ into pCTC-FLAG (Sigma), and expressed the resulting plasmid pCTC-YgfZ in RS218. SDS-PAGE analysis demonstrated that YgfZ is predominantly located in the periplasmic space $(54 \%)$, but it is also detected in the cytoplasmic $(41 \%)$ and inner-membrane $(5 \%)$ fractions (Fig. 5a). As shown in Fig. 5(b), the cytoplasmic fraction contained $\beta$-Gal protein, but was devoid of periplasmic protein PhoA (alkaline phosphatase, AP). In contrast, the periplasmic fraction contained PhoA, but was devoid of $\beta$-Gal, outer-membrane protein OmpA and inner-membrane protein NuoCD, suggesting that our cell fractions exhibited their expected protein profile. The predominant demonstration of YgfZ in the periplasmic space, therefore, is not due to contamination of the periplasmic fraction by cytoplasmic and inner-membrane proteins. 


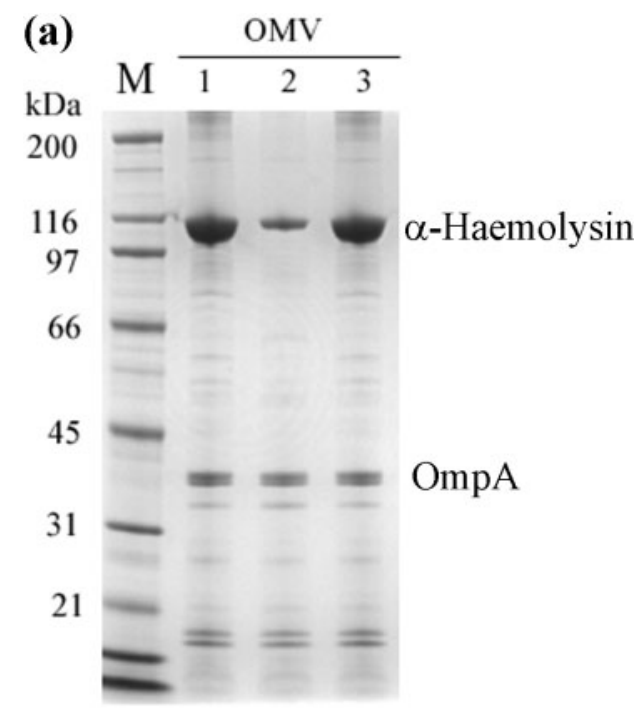

(b)

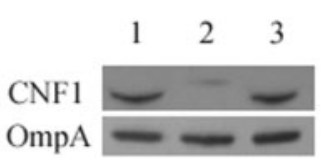

Fig. 4. Complementation of strain $\Delta h n s / \Delta y g f Z$ with $y g f Z$ restores CNF1 secretion. (a) SDS-PAGE analysis of the protein profile in OMVs prepared from $\Delta h n s$ (lane 1), $\Delta h n s / \Delta y g f Z$ (lane 2) and YK16 ( $\Delta h n s / \Delta y g f Z$ mutant complemented with $y g f Z$ ) (lane 3). M, molecular size markers. (b) Demonstration of CNF1 in OMVs isolated from $\Delta h n s$ (lane 1), $\Delta h n s / \Delta y g f Z$ (lane 2) and YK16 ( $\Delta h n s /$ $\Delta y g f Z$ mutant complemented with ygfZ) (lane 3).

\section{YgfZ promotes invasion of HBMEC by E. coli K1}

CNF1 is known to contribute to E. coli K1 invasion of HBMEC (Khan et al., 2002), and we next examined whether YgfZ contributes to the HBMEC invasion by virtue of affecting CNF1 secretion. HBMEC invasion assays revealed that the $\Delta y g f Z$ mutant was significantly defective in invasion of HBMEC compared to the parent strain RS218 (Fig. 6). We then complemented the $\Delta y g f Z$ mutant with $y g f Z$ using Tn7 insertion into the second benign site of the chromosome, resulting in strain YK7. The HBMEC invasion of strain YK7 was restored to the level of the parent strain (Fig. 6).

\section{DISCUSSION}

In this study, we have demonstrated that YgfZ is involved in secretion of CNF1 into OMVs in meningitis-causing E. coli $\mathrm{K} 1$. This is shown by the fact that YgfZ deletion resulted in a decreased amount of CNF1 in the OMVs without affecting the amount of CNF1 expression in the cytoplasm, periplasm and outer membrane, and that the amount of CNF1 was restored to that of the parent strain by complementation with $y g f Z$.

YgfZ is a folate-binding protein (Teplyakov et al., 2004; Waller et al., 2010), but exogenous folate did not enhance CNF1 secretion (data not shown). YgfZ belongs to the COG0354 group, which was suggested to have a conserved, folate-dependent function in the activity of certain $\mathrm{Fe}-\mathrm{S}$ cluster enzymes (Waller et al., 2010). Previously, we

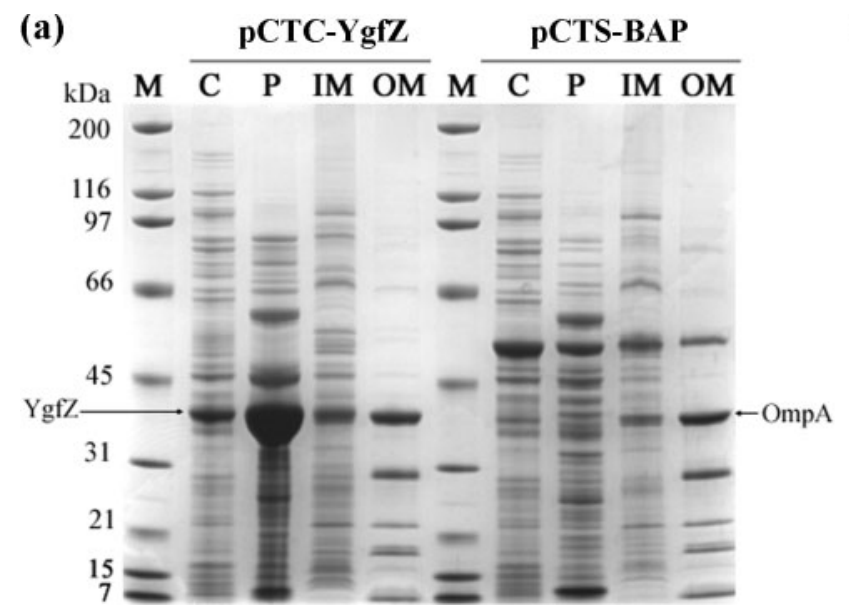

(b)

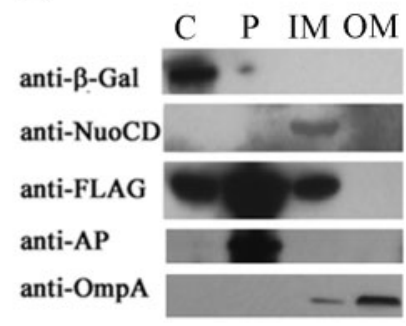

Fig. 5. YgfZ is predominantly localized in the periplasm. (a) SDS-PAGE analysis of the protein profiles of different fractions [cytoplasmic (C), periplasmic (P), inner membrane (IM) and outer membrane (OM)]. The gel was stained with Coomassie blue. pCTC-YgfZ is the expression plasmid of FLAG-tagged YgfZ; pCTS-BAP represents pFLAG-CTS-BAP (Sigma), which is a 6735 bp E. coli plasmid used for efficient and controlled periplasmic expression of C-terminal FLAG-BAP fusion protein. For the $\mathrm{C}$ fraction, $20 \mu \mathrm{g}$ total protein was loaded. For the $\mathrm{P}, \mathrm{IM}$ and $\mathrm{OM}$ fractions, samples were loaded in a proportional manner; the amount of periplasmic protein loaded was equal to the total periplasmic protein that was collected from $3 \times 10^{9}$ bacteria (the number of bacteria was estimated from the $\mathrm{OD}_{620}$ ). $\mathrm{M}$, molecular size markers. (b) Western blot analysis of different fractions of pCTC-ygfZ/RS218 with different antibodies as listed on the left. 


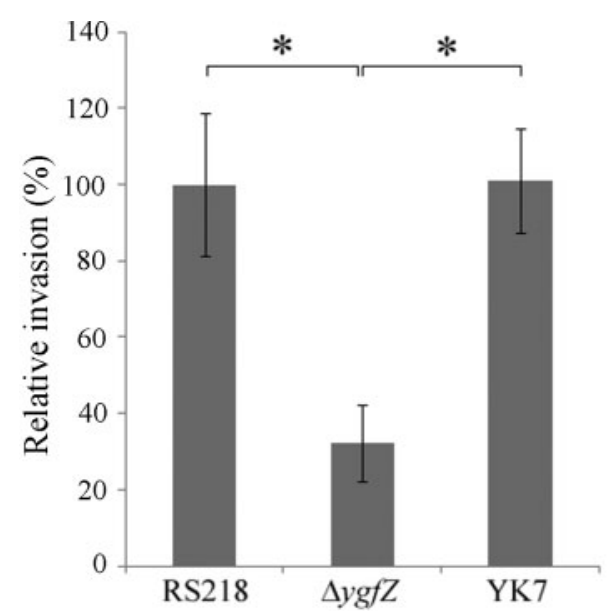

Fig. 6. The $y g f Z$ deletion mutant of $E$. coli strain RS218 exhibits significantly decreased invasion of HBMEC. To determine whether YgfZ plays a role in $E$. coli invasion of HBMEC, invasion assays were performed using the $y g f Z$ deletion mutant and the complemented strain YK7, and the results were expressed as relative invasion frequency (\%) compared to the invasion frequency of wild-type strain RS218, which was set at $100 \%$. ${ }^{*}, P<0.05$. The data represent the mean $\pm S D$ of three independent assays performed in triplicate.

identified that ferredoxin (Fe-S assembly protein) is involved in CNF1 secretion across the bacterial inner membrane (Yu \& Kim, 2010), and comparative genomics studies suggest that COG0354 genes cluster with genes encoding $\mathrm{Fe}-\mathrm{S}$ enzymes or related proteins (Waller et al., 2010). These findings suggest that $\mathrm{Fe}-\mathrm{S}$ protein may be involved with YfgZ in the CNF1 secretion system. This concept is suggested by the demonstration that YgfZ is reported to be in complex with IscA (Hu et al., 2009) and our identification of another transposon mutant of $h s c A$ (located within the iscA operon, downstream of the $f d x$ gene), which is also defective in secretion of CNF1 across the outer membrane (unpublished data). Additional studies are needed to elucidate whether and/or how Fe-S proteins are involved in CNF1 secretion.

We analysed the subcellular distribution of YgfZ, and showed that this protein is predominantly localized in the periplasm (Fig. 5). This is not consistent with previous prediction that YgfZ is a cytoplasmic protein; the discrepancy might stem from our insufficient information on the secretion signal peptide of YgfZ. The folate (vitamin $\mathrm{B}_{9}$ )-binding capability of $\mathrm{YgfZ}$ is reminiscent of the involvement of $\mathrm{BtuB}$ (the receptor of vitamin $\mathrm{B}_{12}$ ) in translocation of colicins, in which colicins bind to BtuB, recruit the porin $\mathrm{OmpF}$ or $\mathrm{OmpC}$ and then traverse the lumen of the $\beta$-barrel of the porin (Housden et al., 2010; James et al., 1996). However, our pull-down experiment did not support the idea that CNF1 may interact with YgfZ (data not shown).

YgfZ was proposed to have a role in one-carbon metabolism, such as transferring $\mathrm{C}_{1}$ units (Teplyakov et al., 2004). Recent studies showed that YgfZ is involved in methylating tRNA and plumbagin (an active ingredient of anti-bacterial herbs) (Chen et al., 2006; Lin et al., 2010; Ote et al., 2006). Another recent study showed that a lipopolysaccharide modification enzyme in Porphyromonas gingivalis contributes to selective sorting of cargo proteins into OMVs (Haurat et al., 2011). These findings prompt us to hypothesize that the deficiency of sorting CNF1 into OMVs in the YgfZ mutant may stem from its failure to modify certain component(s) of the CNF1 secretion machinery. Additional studies on the CNF1 secretion machinery are likely to support and/or disprove this hypothesis.

Despite the availability of crystal structure information on YgfZ (Teplyakov et al., 2004), the working mechanism of this protein is unclear. Our current hypothesis is that YgfZ together with Fe-S assembly complex modifies CNF1 or certain component(s) of the bacteria (e.g. lipopolysaccharide or outer-membrane protein), which can specifically sort CNF1 as a cargo protein of OMVs. Studies are in progress to examine this hypothesis. Further elucidation of the mechanisms involved in the CNF1 secretion network affected by YgfZ is likely to enhance our knowledge of the pathogenesis of E. coli meningitis.

\section{ACKNOWLEDGEMENTS}

This work was supported in part by the NIH (grant NS26310-22). The authors thank Dr Allison O'Brien for providing the CNF1 monoclonal antibody, Dr Nancy L. Craig for providing pGRG36 plasmid, Dr Takao Yagi for providing NuoCD antibody, and Dr Fred Heffron for providing plasmid pMini-Tn5-cycler. We would also like to thank Dr George Niemann for helpful suggestions and discussions.

\section{REFERENCES}

Aldick, T., Bielaszewska, M., Uhlin, B. E., Humpf, H. U., Wai, S. N. \& Karch, H. (2009). Vesicular stabilization and activity augmentation of enterohaemorrhagic Escherichia coli haemolysin. Mol Microbiol 71, 1496-1508.

Boquet, P. (2001). The cytotoxic necrotizing factor 1 (CNF1) from Escherichia coli. Toxicon 39, 1673-1680.

Caprioli, A., Falbo, V., Roda, L. G., Ruggeri, F. M. \& Zona, C. (1983). Partial purification and characterization of an Escherichia coli toxic factor that induces morphological cell alterations. Infect Immun 39, 1300-1306.

Castro-Guerrero, N., Sinha, P. K., Torres-Bacete, J., Matsuno-Yagi, A. \& Yagi, T. (2010). Pivotal roles of three conserved carboxyl residues of the NuoC $(30 \mathrm{k})$ segment in the structural integrity of protontranslocating NADH-quinone oxidoreductase from Escherichia coli. Biochemistry 49, 10072-10080. 
Charpentier, X. \& Oswald, E. (2004). Identification of the secretion and translocation domain of the enteropathogenic and enterohemorrhagic Escherichia coli effector Cif, using TEM-1 $\beta$-lactamase as a new fluorescence-based reporter. J Bacteriol 186, 5486-5495.

Chen, J. W., Sun, C. M., Sheng, W. L., Wang, Y. C. \& Syu, W. J. (2006). Expression analysis of up-regulated genes responding to plumbagin in Escherichia coli. J Bacteriol 188, 456-463.

Chung, J. W., Hong, S. J., Kim, K. J., Goti, D., Stins, M. F., Shin, S., Dawson, V. L., Dawson, T. M. \& Kim, K. S. (2003). 37-kDa laminin receptor precursor modulates cytotoxic necrotizing factor 1-mediated RhoA activation and bacterial uptake. J Biol Chem 278, 16857-16862.

Datsenko, K. A. \& Wanner, B. L. (2000). One-step inactivation of chromosomal genes in Escherichia coli K-12 using PCR products. Proc Natl Acad Sci U S A 97, 6640-6645.

Davis, J. M., Carvalho, H. M., Rasmussen, S. B. \& O'Brien, A. D. (2006). Cytotoxic necrotizing factor type 1 delivered by outer membrane vesicles of uropathogenic Escherichia coli attenuates polymorphonuclear leukocyte antimicrobial activity and chemotaxis. Infect Immun 74, 4401-4408.

Economou, A., Christie, P. J., Fernandez, R. C., Palmer, T., Plano, G. V. \& Pugsley, A. P. (2006). Secretion by numbers: protein traffic in prokaryotes. Mol Microbiol 62, 308-319.

Falzano, L., Fiorentini, C., Donelli, G., Michel, E., Kocks, C., Cossart, P., Cabanié, L., Oswald, E. \& Boquet, P. (1993). Induction of phagocytic behaviour in human epithelial cells by Escherichia coli cytotoxic necrotizing factor type 1. Mol Microbiol 9, 1247-1254.

Flatau, G., Lemichez, E., Gauthier, M., Chardin, P., Paris, S., Fiorentini, C. \& Boquet, P. (1997). Toxin-induced activation of the G protein p21 Rho by deamidation of glutamine. Nature 387, 729733.

Geddes, K., Worley, M., Niemann, G. \& Heffron, F. (2005). Identification of new secreted effectors in Salmonella enterica serovar Typhimurium. Infect Immun 73, 6260-6271.

Haurat, M. F., Aduse-Opoku, J., Rangarajan, M., Dorobantu, L., Gray, M. R., Curtis, M. A. \& Feldman, M. F. (2011). Selective sorting of cargo proteins into bacterial membrane vesicles. J Biol Chem 286, 1269 1276.

Housden, N. G., Wojdyla, J. A., Korczynska, J., Grishkovskaya, I., Kirkpatrick, N., Brzozowski, A. M. \& Kleanthous, C. (2010). Directed epitope delivery across the Escherichia coli outer membrane through the porin OmpF. Proc Natl Acad Sci U S A 107, 21412-21417.

Hu, P., Janga, S. C., Babu, M., Díaz-Mejía, J. J., Butland, G., Yang, W., Pogoutse, O., Guo, X., Phanse, S. \& other authors (2009). Global functional atlas of Escherichia coli encompassing previously uncharacterized proteins. PLoS Biol 7, e96.

James, R., Kleanthous, C. \& Moore, G. R. (1996). The biology of E colicins: paradigms and paradoxes. Microbiology 142, 1569-1580.

Kesty, N. C., Mason, K. M., Reedy, M., Miller, S. E. \& Kuehn, M. J. (2004). Enterotoxigenic Escherichia coli vesicles target toxin delivery into mammalian cells. EMBO J 23, 4538-4549.

Khan, N. A., Wang, Y., Kim, K. J., Chung, J. W., Wass, C. A. \& Kim, K. S. (2002). Cytotoxic necrotizing factor-1 contributes to Escherichia coli K1 invasion of the central nervous system. J Biol Chem 277, 1560715612.

Kim, K. S. (2003). Pathogenesis of bacterial meningitis: from bacteraemia to neuronal injury. Nat Rev Neurosci 4, 376-385.

Kim, K. S. (2008). Mechanisms of microbial traversal of the bloodbrain barrier. Nat Rev Microbiol 6, 625-634.

Kim, K. J., Chung, J. W. \& Kim, K. S. (2005). 67-kDa laminin receptor promotes internalization of cytotoxic necrotizing factor 1-expressing
Escherichia coli K1 into human brain microvascular endothelial cells. J Biol Chem 280, 1360-1368.

Knust, Z. \& Schmidt, G. (2010). Cytotoxic necrotizing factors (CNFs) - a growing toxin family. Toxins (Basel) 2, 116-127.

Kouokam, J. C., Wai, S. N., Fällman, M., Dobrindt, U., Hacker, J. \& Uhlin, B. E. (2006). Active cytotoxic necrotizing factor 1 associated with outer membrane vesicles from uropathogenic Escherichia coli. Infect Immun 74, 2022-2030.

Kume, K., Nakai, T., Samejima, Y. \& Sugimoto, C. (1986). Properties of dermonecrotic toxin prepared from sonic extracts Bordetella bronchiseptica. Infect Immun 52, 370-377.

Landraud, L., Gauthier, M., Fosse, T. \& Boquet, P. (2000). Frequency of Escherichia coli strains producing the cytotoxic necrotizing factor (CNF1) in nosocomial urinary tract infections. Lett Appl Microbiol 30, 213-216.

Lemonnier, M., Landraud, L. \& Lemichez, E. (2007). Rho GTPaseactivating bacterial toxins: from bacterial virulence regulation to eukaryotic cell biology. FEMS Microbiol Rev 31, 515-534.

Lin, C. N., Syu, W. J., Sun, W. S., Chen, J. W., Chen, T. H., Don, M. J. \& Wang, S. H. (2010). A role of $y g f Z$ in the Escherichia coli response to plumbagin challenge. J Biomed Sci 17, 84.

Lockman, H. A., Gillespie, R. A., Baker, B. D. \& Shakhnovich, E. (2002). Yersinia pseudotuberculosis produces a cytotoxic necrotizing factor. Infect Immun 70, 2708-2714.

Mashburn-Warren, L. M. \& Whiteley, M. (2006). Special delivery: vesicle trafficking in prokaryotes. Mol Microbiol 61, 839-846.

Mashburn-Warren, L., Howe, J., Garidel, P., Richter, W., Steiniger, F., Roessle, M., Brandenburg, K. \& Whiteley, M. (2008). Interaction of quorum signals with outer membrane lipids: insights into prokaryotic membrane vesicle formation. Mol Microbiol 69, 491-502.

McKenzie, G. J. \& Craig, N. L. (2006). Fast, easy and efficient: sitespecific insertion of transgenes into enterobacterial chromosomes using $\operatorname{Tn} 7$ without need for selection of the insertion event. BMC Microbiol 6, 39.

Meysick, K. C., Mills, M. \& O'Brien, A. D. (2001). Epitope mapping of monoclonal antibodies capable of neutralizing cytotoxic necrotizing factor type 1 of uropathogenic Escherichia coli. Infect Immun 69, 2066-2074.

Mills, E., Baruch, K., Charpentier, X., Kobi, S. \& Rosenshine, I. (2008). Real-time analysis of effector translocation by the type III secretion system of enteropathogenic Escherichia coli. Cell Host Microbe 3, 104-113.

Ote, T., Hashimoto, M., Ikeuchi, Y., Su'etsugu, M., Suzuki, T., Katayama, T. \& Kato, J. (2006). Involvement of the Escherichia coli folate-binding protein YgfZ in RNA modification and regulation of chromosomal replication initiation. Mol Microbiol 59, 265-275.

Schmidt, G., Sehr, P., Wilm, M., Selzer, J., Mann, M. \& Aktories, K. (1997). Gln 63 of Rho is deamidated by Escherichia coli cytotoxic necrotizing factor-1. Nature 387, 725-729.

Teplyakov, A., Obmolova, G., Sarikaya, E., Pullalarevu, S., Krajewski, W., Galkin, A., Howard, A. J., Herzberg, O. \& Gilliland, G. L. (2004). Crystal structure of the YgfZ protein from Escherichia coli suggests a folatedependent regulatory role in one-carbon metabolism. J Bacteriol 186, 7134-7140.

Vouret-Craviari, V., Grall, D., Flatau, G., Pouysségur, J., Boquet, P. \& Van Obberghen-Schilling, E. (1999). Effects of cytotoxic necrotizing factor 1 and lethal toxin on actin cytoskeleton and VE-cadherin localization in human endothelial cell monolayers. Infect Immun 67, 3002-3008. 
Wai, S. N., Lindmark, B., Söderblom, T., Takade, A., Westermark, M., Oscarsson, J., Jass, J., Richter-Dahlfors, A., Mizunoe, Y. \& Uhlin, B. E. (2003). Vesicle-mediated export and assembly of pore-forming oligomers of the enterobacterial ClyA cytotoxin. Cell 115, 25-35.

Waller, J. C., Alvarez, S., Naponelli, V., Lara-Nuñez, A., Blaby, I. K., Da Silva, V., Ziemak, M. J., Vickers, T. J., Beverley, S. M. \& other authors (2010). A role for tetrahydrofolates in the metabolism of iron-sulfur clusters in all domains of life. Proc Natl Acad Sci U S A 107, 1041210417.
Yu, H. \& Kim, K. S. (2010). Ferredoxin is involved in secretion of cytotoxic necrotizing factor 1 across the cytoplasmic membrane in Escherichia coli $\mathrm{K} 1$. Infect Immun 78, 838-844.

Zlokarnik, G., Negulescu, P. A., Knapp, T. E., Mere, L., Burres, N., Feng, L., Whitney, M., Roemer, K. \& Tsien, R. Y. (1998). Quantitation of transcription and clonal selection of single living cells with $\beta$ lactamase as reporter. Science $\mathbf{2 7 9}, \mathbf{8 4 - 8 8 .}$

Edited by: D. L. Gally 